\title{
Image and Spectrum Image Denoising under the local low Rank Assumption
}

\author{
Jakob Spiegelberg ${ }^{1}$, Juan Carlos Idrobo² and Ján Rusz ${ }^{1}$
}

${ }^{1 .}$ Department of Physics and Astronomy, Uppsala University, Box 516, S-751 20 Uppsala, Sweden

2. Oak Ridge National Laboratory, Center for Nanophase Materials Sciences, Oak Ridge, TN 37831, USA

The signal to noise ratio (SNR) in signals from various measurement techniques, be it imaging or spectrum imaging, are often strongly noise corrupted. Be it due to the beam sensitivity of the sample which does not permit for prolonged exposure times, sample drift issues or also simply the inherent weakness of the signal of interest, there are many possible causes for an insufficiently low SNR in individual spectra (or pixels) for a direct analysis.

Alongside instrumental advances, modern data processing techniques can aid the detection of signals of interest. Modern denoising algorithms can efficiently reduce the noise content in the data at hand enabling their interpretation. The three principal denoising strategies used in state of the art algorithms are the exploitation of (self-)similarity by averaging similar signals (e.g., averaging of two consecutively measured images), truncation of expansion coefficients in some sparsity promoting basis the signal is transformed to (e.g., Fourier filtering), or low rank approximations (e.g., in principal component analysis (PCA)). High-performing algorithms, such as the BM3D method [1] or also NLPCA [2], often combine these concepts to achieve a better denoising result. Similarly, a local, patch based, description of images has been found to be effective for denoising purposes.

In this presentation, we will introduce the recent local low rank (LLR) denoising method [3]. The LLR scheme is applicable to data tensors of any dimension making it straightforwardly applicable to anything from single spectra to images and spectrum images, even with 4 (or more) dimensions. I.e., given a noisy data tensor $X$, a $n$ pixel window is chosen around every channel in a mode which is of high rank so that it can not be compressed (and thus denoised) using a multilinear singular value decomposition (MLSVD, as tensor generalization of PCA, see, e.g., [4]), and subsequently stacked in an additional mode. The thus obtained stacking tensor consists of small and/or compressible modes, so that a good denoising can be achieved by applying MLSVD. The denoised data are then readily obtained by inverting the stacking. E.g., for image denoising, both spatial modes may have high degrees of complexity. A $n$ by $m$ pixel window would be drawn around every pixel, the obtained image patches are then stacked in a three-dimensional tensor, which is denoised using MLSVD.

While the above description of LLR is undeniably dense, it is also complete, which highlights one of the advantages of LLR over previous patch based image denoising algorithms (e.g., BM3D or NLPCA): Apart from the choice of the window size, LLR is a parameter less method (other parameters are estimated from the data), which avoids fine-tuning issues when applied in practice. Furthermore, due to its simplicity, it is straightforward to prove convergence of the algorithm, or to implement it (given that a linear algebra package is at hand).

LLR performs competitively for denoising of both spectrum images and STEM images. We will give examples to either application, demonstrating STEM denoising retaining fine image features such as row wise distortions, as well as fine-structure analysis in EELS boosted by the spatial denoising of LLR. 
Comparisons to previous denoising methods are drawn.

\section{References:}

[1] K. Dabov et al, Image Process. IEEE Trans. 16 (2007), 8.

[2] C. A. Deledalle et al, Proceedings of the British Machine Vision Conference (2011) 25.1-25.10.

[3] J. Spiegelberg et al, Ultramicroscopy 187 (2018), 34-42.

[4] J. Spiegelberg et al, Ultramicroscopy 175 (2017) 36-45.

[5] We thank Wu Zhou, Chinese Academy of Sciences, for supplying the sample used in all the measurement. The authors acknowledge support from the Center of Interdisciplinary Mathematics (CIM) at Uppsala University, the Swedish Research Council, the Göran Gustafsson's Foundation, the K. and A. Wallenberg Foundation (project no. 2015.0060). This research was partially supported by the Center for Nanophase Materials Sciences, which is a DOE Office of Science User Facility.
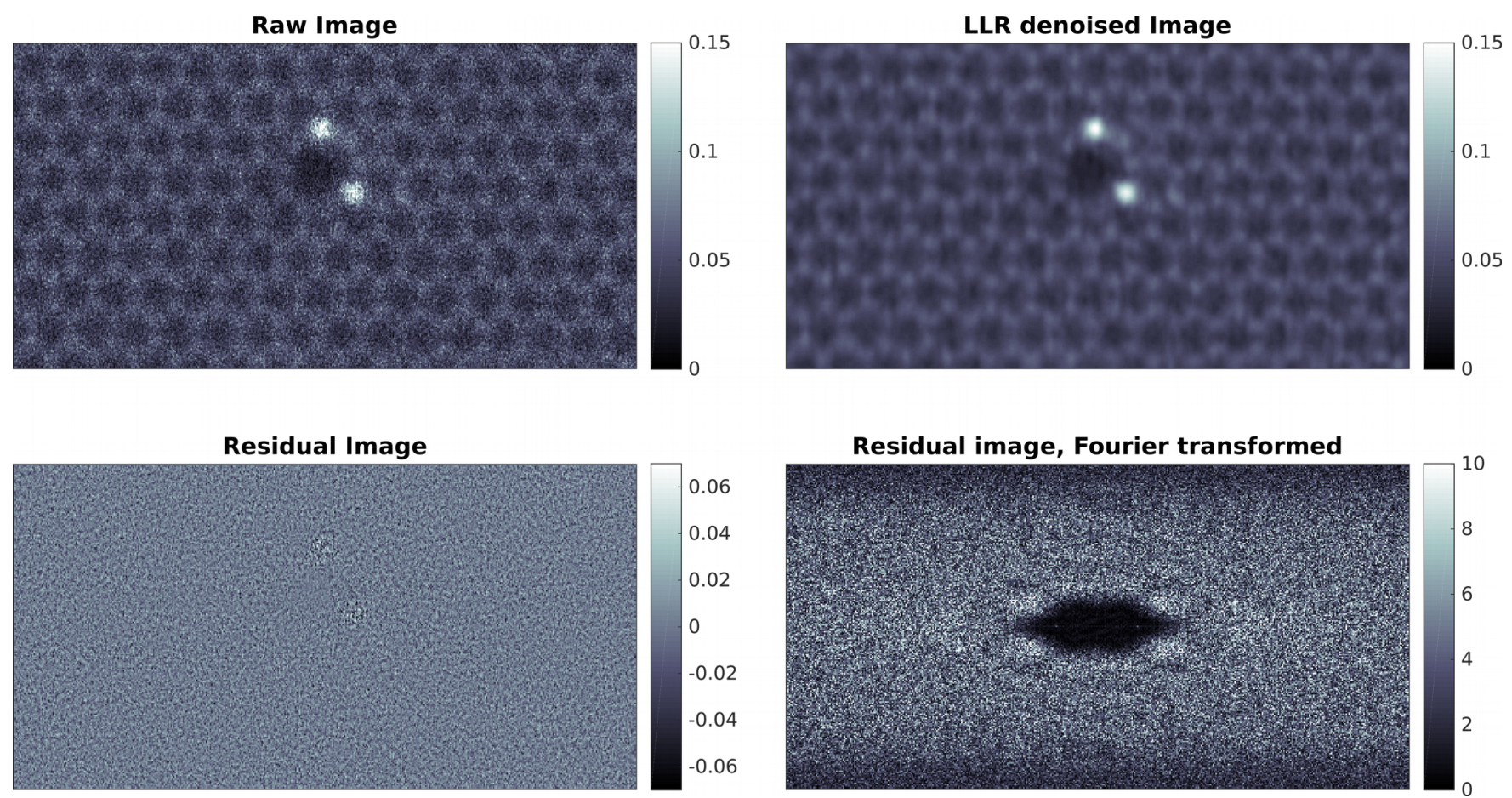

Figure 1. Example of a LLR denoising. An image of a defect in the graphene lattice becomes denoised while retaining minor distortions of the lattice well. In neither residual image can any lattice information be discerned. 